\title{
POLINEUROPATIA PERIFERICA INDUZIDA POR BENZONIDAZOL NO TRATAMENTO DA DOENÇA DE CHAGAS
}

\author{
CARLOS ROBERTO DEFARIA * \\ SEBASTIAO EURICO DE MELO-SOUZA * * \\ ANIS RASSI **
}

Vários estudos neurofisiológicos recentes têm mostrado de forma inequívoca a existência de desnervação motora em pacientes com doença de Chagas aguda e crônica 3,4,6,9. Os achados indicam o corno anterior da medula como o mais provável sítio da lesão primária. Evidências neurofisiológicas que comprovam o envolvimento dos nervos periféricos ainda não foram publicadas, embora vários pacientes apresentem quadro clínico sugestivo de neuropatia periférica e mesmo alguns achados, não conclusivos, demonstrem discreta redução da amplitude de potenciais sensitivos ou da velocidade de condução nervosa sensitiva 5 . Recentemente, pelo método de fibra desfiada ("teasing fibre") foi demonstrada a existência de desmielinização segmentar e degeneração walleriana em fibras nervosas do nervo ciático e do plexo braquial em $100 \%$ dos comundongos experimentalmente infectados pelo $T$. Cruzi, que sobreviveram à fase aguda da infecção 1.

Considerando que várias drogas testadas no tratamento da doença de Chagas são neurotóxicas, decidimos estender nossas pesquisas a pacientes chagásicos virgens de tratamento (VT) e acompanhar do ponto de vista clínico e eletromiográfico o comportamento do sistema nervoso desses pacientes sob ação do benzonidazol. Os pacientes foram observados de 1977 a 1978, sendo controlados por um dos autores (A.R.), examinados neurologicamente por outro (S.E.M-S) e eletrofisiologicamete pelo terceiro (C.R.DeF.) e fez parte de avaliação do benzonidazol 8 , que estava sendo testado antes de sua comercialização, que ocorreu em 1981. A observaçăo de A. Rassi de possiveis efeitos neurotóxicos ensejou este estudo prospectivo.

\section{MATERIAL E METODOS}

o material constou de 53 pacientes em forma crônica da moléstia de Chagas, V'T com imunofluorescência e xenodiagnóstico positivos para a doença: 25 eram homens a 28 mulheres, com idades compreendidas entre 20 e 56 anos (média $38,72 \pm 8,24$ ). Estes pacientes foram divididos em quatro grupos de acordo $\mathrm{com}$ esquema terapêutico: esquema I. $5 \mathrm{mg} / \mathrm{Kg} /$ dia durante 30 dias; esquema II, $5 \mathrm{mg} / \mathrm{Kg} /$ dia durante 60 dias; esquema

Trabalho realizado no Instituto de Neurologia de Goiânia: * Chefe do Departamento de Neurofisiologia Clínica do Instituto de Neurologia de Golânia; * Professor Adjunto de Clínica Médica da. Universidade Federal de Goiás. Os resultados preliminares foram apresentados no Congresso Internacional sobre Doença de Chagas (Rio de Janeiro, 1979). 
III, $3 \mathrm{mg} / \mathrm{Kg} / \mathrm{dia}$ durante 90 dias: esquema IV, $10 \mathrm{mg} / \mathrm{Kg} /$ dia durante 10 dias + $3 \mathrm{mg} / \mathrm{Kg} /$ dia durante 50 dias.

Todos os pacientes foram avaliados clínica e eletromiograficamente, incluindo estudo das velocidades de conduçăo nervosa motora e sensitiva, amplitude dos potenciais sensitivos e contagem de unidades motoras (UM). Estes testes neurofisiológicos foram feitos com equipamento composto de: osciloscópio Tektronix de 3 canais, com memória de tela e persistêncla variável com ganho de até $5_{\mu} \mathrm{V} /$ divisão, resposta de frequència de 0 a 1 MHz; estimulador Dual High Voltage Stimulator-Devices Type 3070; Averager Neurolog ML 750-Devices; digitimer D-100, Devices. As velocidades de conduçăo nervosa foram medidas com eletrodos de superfície sempre no sentido ortodrômico (5). Os eletrodos dos troncos nervosos para estímulo ou registro foram discos de prata $d e \quad 0,5 \mathrm{~cm}$ de diametro, separados por $2 \mathrm{~cm}$ de distância. Os eletrodos musculares foram placas de prata de $6 \mathrm{~cm} \times 0,4 \mathrm{~cm}$, com espessura de $0,2 \mathrm{~mm}$. A técnica de contageri de UM foi a de McComas \& $\mathrm{Col}(\gamma)$. Foram estudadas as fibras sensitivas dos nervos mediano e ulnar, as fibras motoras dos nervos mediano, ulnar e peroneal e foram contadas as unidades motoras dos músculos abductor policis brevis (APB), abductor digiti minimi (ADM) e extensor digitorum brevis ( $\mathrm{EDB})$.

\section{RESULTADOS}

Antes do tratamento - Os pacientes apresentaram nos exames pré-tratamento os resultados que se seguem. Exame neurológico - normal, 23 pacientes (45\%): dor discreta, incomodativa, profunda nos membros inferiores, 11 pacientes (20\%); reflexos abolídos ou assimétricos nos membros inferiores, sem queixas subjetivas, 4 pacientes (7\%); dor discreta, incomodativa, profunda nos membros inferiores + assimetria ou abolição de reflexos nos membros inferiores, 15 pacientes $(28 \%)$. Exame neurofisíológico - APB (53 pacientes): normal 17 (32\%), levemente reduzido 2 , moderadamente reduzido 20, gravemente reduzido 14; ADM (49 pacientes): normal $35(71,5 \%)$, levemente reduzido 7. moderadamente reduzido 6, gravemente reduzido 1; EDB (47 pacientes): normal 27 $(57,5 \%)$, levemente reduzido $\mathbf{1 0}$, moderadamente reduzido 9 , gravemente reduzino 1 . As velociàades de condução nervosa motora e sensitiva, bem como a amplitude de potenciais sensitivos (membros superiores) foram normais em todos os pacientes examinados: nervo mediano (motor, 53 pacientes; sensitivo, 48 pacientes), nervo ulnar (motor, 49 pacientes; sensittvo, 47 pacientes), nervo peroneal (motor, 49 pacientes). A tabela 1 reflete 0 comportamento dos pacientes tratados em relação aos exames pré-tratamento. A perda de UM não é assinalada em relação ao número normal, mas, sim, em relação no número do exame anterior. Referimos esta perda com $I$ para casos inalterados, $R$ para perdas diseretas, RR para perdas moderadas e RRR para perdas acentuadas.

Após tratamento - Dos 53 pacientes pré-selecionados, 24 não foram tratados ou não fizeram controle pós-tratamento. Dos 29 pacientes tratados, os exames de controle revelaram os dados que seguem. Exame neurológico - normal, 13 (44\%); dor discreta, incomodativa, profunda de membros inferiores, $16(55 \%)$; reflexos abolidos ou assimétricos em membros inferiores, 7 (24\%). Exame neurofisiológico - Dezoito dentre os pacientes tratados apresentavam número de $U M$ reduzido no músculo APB no exame prévio, e 12 destes tiveram esta redução de UM aumentada pelo benzonidazol. Dos 11 pacientes com número de unidades motoras normal, apenas três apresentaram redução das unidades do músculo APB após o tratamento. $O$ músculo ADM não spresentou 
alteraçōes significativas com o tratamento, $O$ músculo EDB apresentou redução em 2 de 6 pacientes, dos quais apenas dois tinham número normal no exame prévio (Tabela 1). O benzonidazol provocou redução na velocidade de condução nervosa sensitiva em apenas 4 dos 29 pacientes tratados (Tabela 1).

Um paciente foi submetido pela segunda vez ao esquema terapêtico, tendo desenvolvido polineuropatia periférica grave, com quadro clínico exuberante e acentuada desnervação sensitivo-motora, acompanhada de fibrilação e queda da veloctdade de condução nervosa.

\section{COMENTARIOS}

O propósito deste trabalho foi avaliar a ação do benzonidazol sobre o sistema nervoso periférico, independente de sua ação tripanosomicida. Sinais clínicos de polineuropatia periférica e sua comprovação pelos achados neurofisiológicos foram encontrados em pacientes de todos os grupos, variando em intensidade e proporção, de acordo com o esquema terapêutico utilizado. As alterações clinicas caracterizam-se em sua maioria, por manifestações subjetivas de dor e parestesias, com raros casos de redução objetiva da sensibilidade superficial. Neurofisiologicamente o achado positivo mais comum foi a redução do número de UM aparentemente agravando o quadro dos pacientes já comprometidos. O grupo 2 foi o que apresentou danos mais graves, tanto em relação ao numero de pacientes quanto à intensidade de alterações, tendo $44 \%$ dos pacientes apresentado polineuropatia clínica e $78 \%$, perda de unidades motoras. O grupo 3 apresentou alterações clínicas em $14 \%$ dos pacientes e alterações neurofisiológicas em $43 \%$, tendo sido o segundo em importância. Os grupos 1 e 4 apresentaram reduzido número de alteraçōes neurofisiológicas ( $33 \%$ e $25 \%$ respectivamente) e o grupo 1 teve alguma sintomatologia clinica em $11 \%$ dos pacientes. A velocidade de condução nervosa sensitiva estava reduzida em $14 \%$ dos pacientes (4 pacientes), sendo três dos grupos 2 e 3 (10\%) e um do grupo 4 .

Estes achados evidenciam ser o benzonidazol produto neurotóxico para o homem, provocando polineuropatia periférica dose-dependente, possivelmente com efeito cumulativo. As lesões dos nervos periféricos são provavelmente do tipo axonal ou por desmielinização segmentar de pequena quantidade de fibras finas como evidencia a desnervação motora importante, com preservação da velocidade de condução nervosa motora. Esses dados sugerem também a possibilidade de lesão de células do corno anterior da medula, provavelmente agravando aqueles pacientes já parcialmente afetados pela própria tripanosomíase (Tabela 1). Discreta desmielinização de fibras sensitivas é evidenciada pela redução da velocidade de condução nervosa sensitiva. Também pode-se tratar do comprometimento de fibras finas sem repercussão na velocidade de condução nervosa global do nervo como ocorre na neuropatia diabética sensitiva 2 . Essas suposições são encorajadas pelos achados da paciente M.P.M. que, durante o segundo tratamento com benzonidazol, desenvolveu polineuropatia periférica grave, com quadro clínico exuberante e grosseiras alterações das velocidades de condução nervosa sensitiva e motora, além de acentuada desnervação motora com evidente atividade espontânea ao EMG de agulha. 


\begin{tabular}{|c|c|c|c|c|c|c|c|c|}
\hline \multicolumn{2}{|c|}{ Pacientes } & \multicolumn{4}{|c|}{ Eletromiografia } & \multicolumn{3}{|c|}{$\begin{array}{c}\text { Resultados em \% } \\
\text { de pacientes }\end{array}$} \\
\hline \multirow{2}{*}{ Grupo } & \multirow{2}{*}{ Nome } & \multicolumn{2}{|c|}{ No de UM } & \multicolumn{2}{|c|}{ VCNS } & \multirow{2}{*}{$\begin{array}{l}\text { Red. } \\
\text { de UM }\end{array}$} & \multirow{2}{*}{$\begin{array}{l}\text { Red, } \\
\text { vCNS }\end{array}$} & \multirow{2}{*}{$\begin{array}{l}\text { PNP } \\
\text { ciĺnica }\end{array}$} \\
\hline & & $\mathrm{APB}$ & EDB & MED & ULN & & & \\
\hline I & AFT & I & I & $\mathbf{N}$ & $\mathbf{N}$ & & & \\
\hline Benzonidazol & IRS & $\mathbf{R}$ & $\mathbf{I}$ & $\mathbf{N}$ & $\mathrm{N}$ & & & \\
\hline Total & ILS & RRR & I & $\mathbf{N}$ & $N$ & & & \\
\hline \multirow[t]{6}{*}{$150 \mathrm{mg} / \mathrm{Kg}$} & JI. & I & $\mathbf{I}$ & $\mathrm{N}$ & $\mathrm{N}$ & & & \\
\hline & JRS & I & & $\mathrm{N}$ & $\mathrm{N}$ & & & \\
\hline & $\mathrm{LF}$ & I & & $\mathbf{N}$ & $\mathbf{N}$ & & & \\
\hline & LMF & $R R$ & & $\mathrm{~N}$ & $\mathbf{N}$ & & & \\
\hline & LBP & I & & $\mathbf{N}$ & $\mathbf{N}$ & & & \\
\hline & MRS & $\mathbf{I}$ & & $\mathbf{N}$ & $N$ & $33 \%$ & $0 \%$ & $11 \%$ \\
\hline II & JAV & $\mathrm{RRR}$ & $\mathrm{RR}$ & $\mathbf{R}$ & R & & & \\
\hline Benzonidazol & JN & $\mathrm{RR}$ & & $\mathrm{N}$ & $\mathbf{N}$ & & & \\
\hline Total & LAV & RRR & I & $\mathbf{R}$ & $\mathrm{R}$ & & & \\
\hline \multirow[t]{6}{*}{$300 \mathrm{mg} / \mathrm{Kg}$} & MRA & RRR & I & $\mathrm{N}$ & $\mathbf{N}$ & & & \\
\hline & NLR & $\mathrm{RRR}$ & I & $\mathbf{N}$ & $\mathrm{N}$ & & & \\
\hline & RFS & $\mathrm{R}$ & I & $\mathrm{N}$ & $\mathrm{N}$ & & & \\
\hline & RMA & I & $\mathbf{R R}$ & $\mathbf{N}$ & $\mathrm{N}$ & & & \\
\hline & IOM & $\mathbf{R}$ & $\mathbf{I}$ & $\mathrm{N}$ & $\mathrm{N}$ & & & \\
\hline & AAR & I & I & $\mathbf{N}$ & $\mathrm{N}$ & $78 \%$ & $22 \%$ & $44 \%$ \\
\hline III & APS & $\mathbf{R R R}$ & I & $\mathbf{R}$ & $\mathrm{R}$ & & & \\
\hline Benzonidazol & $\mathrm{DCM}$ & I & I & $N$ & $\mathbf{N}$ & & & \\
\hline Total & GRC & I & I & $\mathrm{N}$ & $\mathrm{N}$ & & & \\
\hline \multirow[t]{4}{*}{$270 \mathrm{mgK} / \mathrm{g}$} & UDS & $\mathrm{N}$ & I & $\mathrm{N}$ & $N$ & & & \\
\hline & FOL & $\mathrm{RR}$ & I & $\mathbf{N}$ & $\mathrm{N}$ & & & \\
\hline & MBG & 1 & & $\mathrm{~N}$ & $\mathrm{~N}$ & & & \\
\hline & FSM & RRR & & $\mathrm{N}$ & $\mathrm{N}$ & $43 \%$ & $14 \%$ & $14 \%$ \\
\hline IV & EGR & I & & $\mathbf{N}$ & $\mathbf{N}$ & & & \\
\hline Benzonidazol & JNS & RRR & & $\mathrm{R}$ & $\mathbf{N}$ & & & \\
\hline Total & EPN & I & & $\mathbf{N}$ & $\mathrm{N}$ & & & \\
\hline $250 \mathrm{mg} / \mathrm{Kg}$ & GGS & I & I & $\mathbf{N}$ & $\mathbf{N}$ & $25 \%$ & $25 \%$ & $0 \%$ \\
\hline
\end{tabular}

Tabela 1 - Controle eletroclinico pos-iratamento: Para pacientes indicados (iniciais do nome) $e$ distribuidos segundo grupos (I, que recebeu $150 \mathrm{mg} / \mathrm{Kg}, I I \quad 300 \mathrm{mg} / \mathrm{Kg}$, III $270 \mathrm{mg} / \mathrm{Kg}, I V \quad 250 \mathrm{mg} / \mathrm{Kg}$ ) em relacão as achados arotes do tratamento por Benzonidazol, indicam-se: os aspectos eletromiograficos quanto ao número de unidades motoras (UM) inalterado (I), discretamente reduzido (menos de 20\%) $R$, moderadamente reduzido (até $40 \%$ ) $R R$ e acentuadamente reduzido (além de $40 \%$ ) RRR nos músculos Abductor Policis Brevis (APB) e Extensor Digitorum Brevis (EDB) e a velocidade de conduça sensitiva (VCNS) cionforme fosse normal (N) ou reduzida ( $R$ ) para os nervos mediano (MED) e ulnar (ULN). Os resultados em \% indicam a quantidade de pacientes que apresentaram perda de unidades motoras (UM), reduça na velocidade de conduça no nervosa sensitiva (VCNS) e instalasão de polineuropatia periferica ( $P$ NP) clinicamente constatada, em relagão ao estado do paciente antes do tratamento. 
A suspensão da droga sucedeu acentuada melhora clinica e normalização das velocidades de condução nervosa, na maioria dos pacientes, embora alguns Celes permanecessem com sequelas após vários meses de uso da droga. Esses pacientes estāo sendo reavaliados quanto aos dados referentes à evolução a longo prazo.

\section{RESUMO}

O estudo evolutivo de pacientes na fase crônica da doença de Chagas, feito antes e após o îratamento experimental com benzonidazol, evidenciou ser esta droga tóxica para o sistema nervoso periférico, dose dependente, com possivel efeito cumulativo en vários pacientes. A polineuropatia foi predominantemente do tipo axonal e mais severa nos pacientes já com evidências neurofisiológicas de desnervação periférica antes do tratamento.

\section{SUMMARY}

Peripheral polineuropathy induced by benzonidazol in the treatment of Chagas' disease.

Neurophysiological exanination before and after the administration of benzonidazol, has shown peripheral polineuropathy induced by this drug in most of the patients treated for chronic Chagas' disease. The polineuropathy was mostly axonal and it was dose dependent being more severe in patients who had desnervation of skeletal muscles before receiving the drug.

\section{REFERENCIAS}

1. BARREIRA, A.A.; SAID, G. \& KRETTLI, A.V. - Multifocal demyelinative lesions of peripheral nerves in experimental chronic Chagas disease. Trans. royal Soc. trop. Med. Hyg. 5: 751, 1981.

2. BROWN, M.I.: MARTINS, J.R. \& ASBURY, A.K. - Painful diabetic neurophaty. Arch. Neurol. 3: 164, 1976.

3. DeFARIA, C.R.: MELO-SOUZA, S.E. de \& RASSI, A. - Motor denervation in Chagas' disease. Abstracts IV th International Congress on Neuromuscular Diseases, Montreal, 1970, abstract 147.

4. DeFARIA, C.R.; MELO-SOUZA, S.E. de \& RASSI, A. - Evidências eletromiogräficas de desnervação motora em pacientes na fase crônica da doença de Chagas. Rev. goiana Med. 25: 125, 1979.

5. DefARIA, C.R.; MELO-SOUZA, S.E. de; RASSI, A. - Estudo neurofisiológico na doença de Chagas. Rev. goiana Med. 28: 3, 1982.

6. DeFARIA, C.R.; MELO-SOUZA, S.E, de; RASSI, A. \& LIMA, A.F. - Evidências eletromiográficas de desnervaçāo motora em pacientes na fase aguda da doença de Chagas. Rev. goiana Med. 25: 153, 1979.

7. McCOMAS, A.J.; FAWCET, P.R.W.; CAMPBELL, M.J. \& SICA, R.E.P. - Electrophysiological estimation of the number of motor units within a human muscle. J. Neurol. Neurosurg \& Psychiat. 34: 121, 1971.

8. MELO-SOUZA, S.E. de; DeFARIA, C.R.; RASSI, A. \& LIMA, A.F. - Polineuropatia com benzonidazol no tratamento da doença de Chagas. Anais do Congresso Internacional sobre Doenca de Chagas. Rio de Janeiro, 1979, resumo, pg. 146.

9. PAGANO, M.A.; ARISTIMUNO, G.C.; COLOMBI, A. \& SICA, R.E.P. - Eletromyographycal findings in human chronic Chagas's disease. Arq. Neuro-Psiquiat. (São Paulo) 36: 316, 1978.

Instituto de Neurologia de Goiania - Caixa Postal 15\% - 74000-Goiania, Go - Brasil. 